\title{
Pathogenesis of chronic obstructive pulmonary disease
}

\author{
Rubin M. Tuder ${ }^{1}$ and Irina Petrache ${ }^{2,3}$ \\ 1Program in Translational Lung Research, Division of Pulmonary Sciences and Critical Care Medicine, Department of Medicine, University of Colorado, \\ School of Medicine, Denver, Colorado, USA. ${ }^{2}$ Division of Pulmonary and Critical Care Medicine, Department of Medicine, and \\ ${ }^{3}$ Richard L. Roudebush Veteran Affairs Medical Center, Indiana University, Indianapolis, Indiana.
}

\begin{abstract}
The current epidemic of chronic obstructive pulmonary disease (COPD) has produced a worldwide health care burden, approaching that imposed by transmittable infectious diseases. COPD is a multidimensional disease, with varied intermediate and clinical phenotypes. This Review discusses the pathogenesis of COPD, with particular focus on emphysema, based on the concept that pulmonary injury involves stages of initiation (by exposure to cigarette smoke, pollutants, and infectious agents), progression, and consolidation. Tissue damage entails complex interactions among oxidative stress, inflammation, extracellular matrix proteolysis, and apoptotic and autophagic cell death. Lung damage by cigarette smoke ultimately leads to self-propagating processes, resulting in macromolecular and structural alterations - features similar to those seen in aging.
\end{abstract}

Tobacco-related diseases, including chronic obstructive pulmonary disease (COPD), account for $3.7 \%$ of the world burden of disability-adjusted life-years (DALYs), a measure of lost years of healthy life (1). Tobacco use, excessive alcohol consumption, and unhealthy diets and physical inactivity contribute to most preventable non-communicable diseases. These diseases are projected to impose a worldwide burden of $\$ 47$ trillion health dollars by 2030 . In contrast, it costs only $\$ 0.40$ per individual per year to implement a program aimed at averting tobacco-related diseases that has the potential to save 25-30 million DALYs (1). Notwithstanding its preventable nature, the increasing prevalence, impact as the third leading cause death in the United States since 2008, and socioeconomic costs (1) call for vigorous research efforts to improve the understanding and, ultimately, management of COPD.

Under the umbrella definition of "decreased airflow that is not fully reversible" classically measured by the forced expiratory volume in one second $\left(\mathrm{FEV}_{1}\right), \mathrm{COPD}$ has a spectrum of clinical presentations, which affects accurate diagnostic phenotyping of patients as well as the design and validation of effective therapies (2). The typical clinical manifestations of the COPD syndrome include chronic bronchitis, a condition of large-airway inflammation and remodeling, and emphysema, a disease of the distal airways and lung parenchyma that manifests as loss of surface area for gas exchange. COPD decreases patients' quality of life due to shortness of breath and chronic productive cough, which can progress over years to chronic hypoxemic and/or hypercarbic respiratory failure. Furthermore, systemic manifestations of COPD such as systemic inflammation, alterations of metabolism, cardiovascular events, and cancer contribute to the untimely death of these patients.

This Review emphasizes recent pathogenetic insights and emerging investigations into the complex and chronic nature of COPD (Table 1). These efforts have the added benefit of providing a window into lung biology, with a broader impact in the understanding of other non-tobacco-related pulmonary diseases.

Conflict of interest: The authors have declared that no conflict of interest exists. Citation for this article: J Clin Invest. 2012;122(8):2749-2755. doi:10.1172/JCI60324.

\section{Initiation}

Tobacco smoke remains the key cause of COPD worldwide. Given that cigarette smoke contains thousands of injurious agents (3), its pathogenicity cannot be stringently studied one compound at a time. Aside from nicotine, heavy metals, and carcinogens, tobacco smoke leads to a significant exposure to oxidants. These include alkyl, alkoyl, and peroxyl organic free radicals (causing lipid peroxidation), $\alpha, \beta$-unsaturated aldehydes (such as acrolein and crotonaldehyde, which cause protein carbonylation and loss of sulfhydryls), and superoxide, $\mathrm{N}_{2} \mathrm{O}$, and nitric oxide (which can generate peroxynitrite, leading to formation of dityrosine and/or 3-nitrotyrosine) (4).

While most studies have addressed medium- to long-term organismal responses to cigarette smoke, insights into immediate host responses to the inhalation of the toxic and oxidant components of smoke have been limited (Figure 1). Both in humans (5) and in rodents (6), tobacco smoke causes airway inflammatory responses within minutes or hours of exposure. One of the earliest manifestations is a breach in the vascular and airway barrier function (7), with brisk recruitment of circulating inflammatory cells to the lung (8). Indeed, oxidants present in the cigarette smoke trigger NF-KB-dependent inflammatory responses (9). The acute inflammatory response appears to be transient in nature and mediated by NF- $\mathrm{KB}$, likely counteracted by regu-

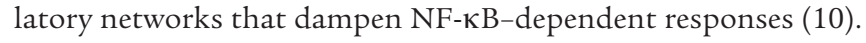
Paradoxically, NF- $\kappa \mathrm{B}$ may also participate in protection against cigarette smoke, as loss of function of the NF-кB p50 subunit augments cigarette smoke inflammatory responses (11).

Recent studies have implicated the host's responses in the augmentation of lung injury by cigarette smoke. For example, cigarette smoke activates inducible nitric oxide synthase, leading to generation of oxidants, such as peroxynitrite $\left(\mathrm{ONOO}^{-}\right)$, which has been linked to alveolar injury due to cigarette smoke (12). Another endogenous mediator of cell injury and inflammation co-opted in the early lung responses to cigarette smoke is the collagen degradation product proline-glycine-proline peptide (PGP), which engages CXCR2 receptors of neutrophils, therefore amplifying initial cigarette smoke-induced inflammation (13). In addition, the LPS in cigarettes may activate TLR4-expressing cells in the lung, leading to the activation of NF- $\kappa \mathrm{B}$ responses (14). However, the 
Table 1

Levels of COPD complexity

\section{Initiation Progression Consolidation}

\section{Level 1: Environment}

Infectious agents

Pollutants

Tobacco

Level 2: Genes

CHRNA3/5

FAM13A

HHIP

SERPIN2

XRCC5

Level 3: Clinical phenotypes

Cancer

Chronic bronchitis

CVD

Depression

Emphysema

Exacerbations

Metabolic syndrome

Osteoporosis

Weight loss

Level 4: Biomarkers

CRP

Endothelial cell microparticles

Exhaled condensate

Level 5: Treatment

Bronchodilators

Inhaled corticosteroids

PDE4 inhibitors

Environmental etiologic factors, genetic basis, clinical phenotypes, biomarkers, and available treatments are listed. The pathogenetic stages of initiation, progression, and consolidation integrate these multiple levels, allowing for a comprehensive approach to the understanding of COPD. CRP, C-reactive protein. Data are from the American Journal of Respiratory and Clinical Care Medicine (103).

role of TLR4 is complex and potentially paradoxical; its absence led to spontaneous emphysema in knockout mice, which showed increased oxidant generation in pulmonary capillary endothelial cells via activation of NADPH oxidase 3 (15).

Recent investigations revealed that organismal sensors of stress might control how early lung responses control subsequent injury to cigarette smoke. RTP801 (encoded by the DNA damage-inducible transcript 4 [Ddit4] gene) is a stress-induced molecule (16) that mediates apoptosis and increases oxidative stress (17) while inhibiting cell growth and proliferation by blocking mTOR signaling (18). Cigarette smoke increased lung RTP801 expression, largely in type II epithelial cells, where it was both necessary and sufficient for NF- $\mathrm{KB}$ activation (6). Mice lacking RTP801 were completely protected against cigarette smoke-induced acute inflammation, apoptosis, and most importantly, against emphysema development following 6 months of exposure (6). These findings indicate a key role of this early stress-response protein and especially its inhibitory effect on the mTOR pathway, evoking a potential therapeutic role for modulation of $\mathrm{mTOR}$ signaling in modifying the early response to cigarette smoke exposure (6).
The master antioxidant transcription factor Nrf2 has been recently implicated in a broad range of lung responses involved in both the initiation and progression of lung injury due to cigarette smoke. Nrf2 controls more than 100 genes involved in antioxidant defenses, detoxification, and cellular physiology (19). Mice lacking Nrf2 show increased susceptibility to lung inflammation by acute cigarette smoke exposure, have upregulated RTP801 expression, and with chronic exposure also show increased susceptibility to alveolar cell apoptosis and development of emphysema (20).

\section{Progression}

The stage of progression of alveolar injury has attracted most of the research efforts in the COPD field (Figure 1). For more than 30 years, initiation and progression have been linked to extracellular matrix proteolysis, notably degradation of elastin by elastases, largely of inflammatory cell source. Key to this paradigm were the landmark discoveries of emphysema in $\alpha 1$-antitrypsin-deficient patients (21) and the induction of emphysema by intratracheal instillation of pancreatic elastase (22), as well as the finding that MMP-12deficient mice are resistant to cigarette smoke-induced mouse emphysema (23). While extracellular matrix proteolysis is a central event in emphysema, it is apparent that it cannot explain the complexity of alveolar destruction in COPD.

A more complex picture of the mechanisms of alveolar destruction leading to emphysema has emerged in the past 12 years. Underlying the discovery of alternative molecular determinants and destructive processes was the important yet straightforward concept that the lung requires ongoing maintenance of its structures, notably during injury $(24,25)$. Variability in an individual's ability to maintain lung structure and promote repair may explain the $20 \%-25 \%$ risk that smokers have of developing COPD as well as the reason that patients differ greatly in their clinical phenotypes and disease severity and progression. This concept has been verified in multiple models of emphysema (26) and, albeit at a descriptive level, supported by studies in lung samples from humans with COPD that demonstrate altered expression of multiple trophic/ maintenance factors, including VEGF (27), Wnt signaling components (28), and adiponectin (29). This concept may also apply to the underlying mechanisms leading to the recently described disappearance of terminal airways in COPD (30).

We believe that mechanisms involved in the progression stage of COPD may be distinctly engaged in generating variable intermediate and clinically relevant disease phenotypes, such as emphysema, chronic airway disease (including chronic bronchitis and bronchiolitis), and systemic disease.

Emphysema. In addition to enhanced lung elastolysis, the failure of the lung maintenance program in the parenchyma distal to the terminal bronchiole leads to a loss of alveolar cells by apoptosis in emphysema. The role of apoptosis was directly tested in mouse models of emphysema induced by a loss of VEGF function; in these models, caspase inhibitors preserved the integrity of alveolar septae $(27,31)$. Fueled by the identification of increased apoptotic cells in the parenchyma of human emphysema lungs (32), multiple mechanistic studies of the drivers and downstream consequences of this form of regulated cell death have emerged. Although it remains disputed which structural cell of the alveolus directs the process of alveolar destruction, it has been established that (a) apoptosis of both epithelial and endothelial cells occurs in models of emphysema $(20,27,33)$, (b) direct instillation or overexpression in the lung of apoptotic effector molecules induces transient 
A

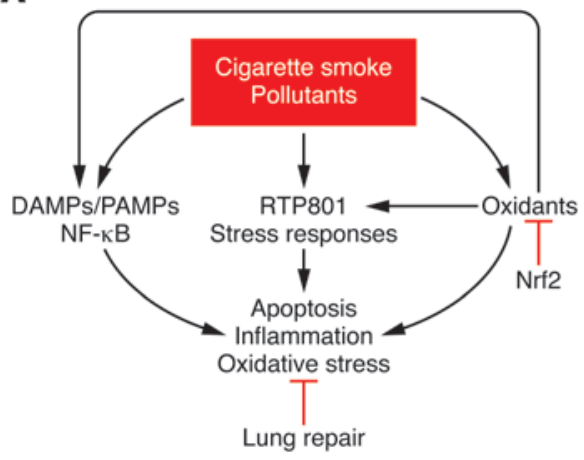

B

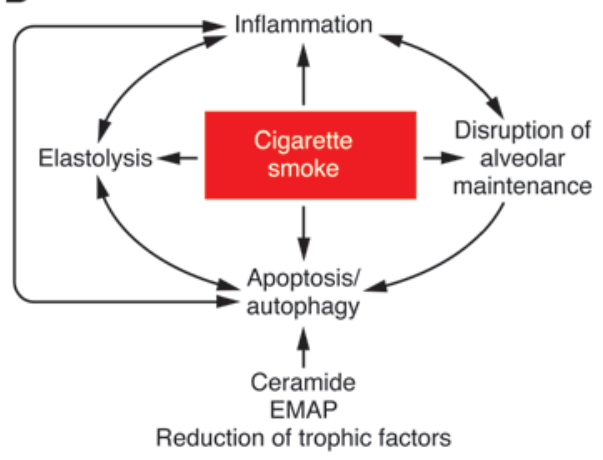

C

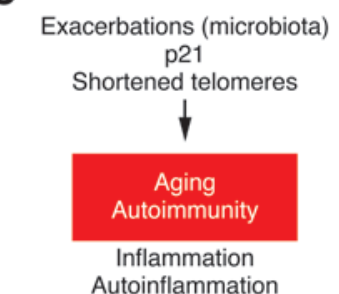

(antielastin T cells, inflammasome NK cells, Th17)

\section{Figure 1}

Pathogenetic factors organized based on their role in the initiation, progression, and consolidation of emphysema. (A) Initiation: Environmental agents trigger host cell responses, largely dominated by inflammation and oxidative stress. RTP801 is activated by cigarette smoke, largely due to oxidants, mediating inflammatory responses, oxidative stress, and alveolar cell death. DAMPs and PAMPs present in tobacco or generated endogenously may further enhance pathologic responses. Nrf2, by activating a host of antioxidant mediators, protects the lung and may promote lung repair processes. (B) Progression: Cigarette smoke disrupts alveolar maintenance, triggering apoptosis and autophagy; moreover, oxidants in tobacco and activated inflammatory and alveolar cells lead to extracellular matrix proteolysis, which further enhances inflammation and promote a feedback loop with apoptosis. Several of these interactions are facilitated by decreased expression of trophic/maintenance factors and endogenous mediators of alveolar destruction, including ceramide and EMAPII. (C) Consolidation: Over decades of exposure to cigarette smoke and endogenous amplifiers of destructive processes, there is progressive lung aging, with autoinflammatory stimuli generated through self-antigens or microbial/viral agents. TH17-positive cells, which are increased in COPD patients, may mediate the autoimmunity process. Macromolecular

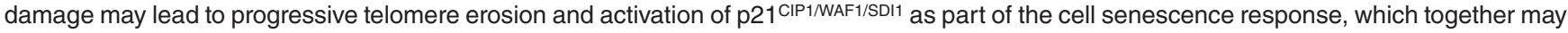
lead to a terminally injured lung.

airspace enlargement (33-36), and (c) specific induction of lung microvascular endothelial cell apoptosis is sufficient to cause a phenotype reminiscent of cigarette smoke-induced emphysema, including influx of inflammatory cells (37).

The induction of cell death in structural cells of the lung parenchyma (epithelial, endothelial, and possibly septal fibroblast cells) in response to cigarette smoke may be related to a loss of growth factors, oxidative stress injury, or intracellular response to stress imposed by noxious exposures (e.g., ER stress, ref. 38; or DNA damage, ref. 39). The ultimate outcome of these processes may differ depending on cell type; in contrast to small airway or alveolar epithelial or endothelial cells, alveolar macrophages are significantly more resistant to the apoptotic effect of direct cigarette smoke exposure or pro-apoptotic ceramides $(40,41)$, largely attributed to increased Akt signaling and increased ceramidase activity (42). Moreover, lung cells may respond to injury by activating pro-survival mechanisms such as autophagy, a response typically triggered by starvation. Lung epithelial cells (43), endothelial cells (44), fibroblasts (45), and alveolar macrophages $(40,45)$ all respond to cigarette smoke exposure by initiating autophagy signaling. Current evidence suggests that the abnormal persistence of such signaling or the inability to complete a physiological autophagic program may increase cellular stress such as ER stress, leading to caspase activation and apoptosis in diseased lungs $(40,44,46)$.

Investigation into the interactions between upstream and downstream events related to alveolar cell apoptosis during emphysema onset and progression led to the key finding of self-amplifying injury loops involving apoptosis, oxidative stress, and inflammation (3-5). This concept may explain the progression of disease despite cessation of exposure to harmful initiators such as cigarette smoking (47). Paradigmatic of this interaction is the upregulation of pro-apoptotic sphingolipids in alveolar cells, including ceramides. Ceramides, which are induced directly by cigarette smoke or indirectly due to VEGF deprivation or oxidative stress, cause apoptosis of alveolar structural cells, self-amplify their own synthesis in a paracrine manner, increase oxidative stress, cause inflammation with activation of extracellular matrix proteases, and impair the clearance of apoptotic cells by lung alveolar macrophages $(33,35$, 41,48 ). Executioner caspases such as caspase- 3 and elastases can in turn proteolytically activate endothelial monocyte-activating protein II (EMAPII), which has a dual action in the lung, causing endothelial cell caspase-dependent apoptosis as well as inflammation via CXCR3-dependent monocyte chemoattraction and activation (36). Additional positive interactions exist between extracellular matrix proteases, such as between cathepsin $\mathrm{S}$ and alveolar cell apoptosis (49), and between alveolar cell apoptosis and oxidative stress (50).

There is growing evidence that pulmonary and systemic inflammation, key events in COPD, may change in nature as the disease progresses. As mentioned above, inflammatory cell activation and influx may be operated by different mechanisms in the later phases of disease, when pathogen-associated molecular patterndriven (PAMP-driven) processes (see below), abnormal apoptotic homeostasis (an apoptotic rate greater than the rate of clearance of dead cells by efferocytosis; ref. 51), and autoimmune responses become more prominently involved in inflammation $(52,53)$. Epigenetic dysregulation may contribute to excessive activation of proinflammatory cytokines and chemokines and steroid resistance $(54,55)$. As the lung maintenance program is eroded during chronic smoke exposure, inflammatory cells, including neutrophils, macrophages, and lymphocytes may change their phenotype. For instance, macrophages may switch toward an M2 phenotype, potentially limiting inflammation yet enhancing fibrosis and weakening their antibacterial functions (56). The concept of evolving inflammatory phenotype in the course of COPD is also supported by studies of lymphocyte involvement in the disease. 
Different populations of lymphocytes may aid in alveolar destruction; the presence of CD8 cells has been associated with disease severity (57), and oligoclonal CD4 cells have been detected in advanced COPD lungs (58), suggesting their participation in autoimmune responses in the consolidation stage of COPD. NK cells, which also accumulate in increased numbers in COPD lungs, may drive alveolar cell apoptosis via crosstalk with corresponding lung epithelial cell NKGD2 ligands (59).

In addition, bacterial and viral infections, common causes of exacerbation in COPD patients and a major cause of pulmonary deterioration and mortality, may profoundly affect the nature of the immune response in the lung. Infectious agents, via PAMPs, may engage specific receptors, initiating host responses that lead to amplification of alveolar injury. Dysfunction of Nrf2 signaling in COPD patients (60) may compromise bacteria clearance (61). Influenza virus, a common cause of acute exacerbations, may synergize with cigarette smoke-induced responses via activation of the retinoic acid inducible gene-1 (RIG-1) helicase system. Indeed, double-stranded RNA viruses (e.g., influenza virus) activate innate signaling, culminating in the activation of interferon regulatory factor- 3 and -7 (IRF- 3 and -7 ) and the subsequent induction of antiviral genes, including NF-кB. This process was elegantly described by studies using the double-stranded RNA mimetic, polyinosinic-polycytidylic acid (poly[I:C]) (62). Poly(I:C) cooperates with cigarette smoke to activate the RIG-1/ MAVS/PKR/IL-18 pathway, causing excessive alveolar cell death, inflammation, and hastened and more extensive emphysema (62). These pathways may interact with endogenous danger-associated molecular patterns (DAMPs), such as high-mobility group box 1 , to amplify early cigarette smoke-induced responses (63). Even elastin dissolution into chemotactic peptides during alveolar destruction further augments inflammatory responses independently of ongoing tobacco exposure (64).

Airway disease. Large and small ( $<2 \mathrm{~mm}$ diameter) airways are central sites of disease in COPD. While large airways undergo chronic inflammation with excessive mucus production, smaller airways are surrounded by inflammatory cells and wall fibrosis and exhibit intraluminal mucus accumulation (65). The discovery that cigarette smoke directly inhibits the CFTR function (66) provides a mechanistic link between smoking and abnormal mucous secretion by airway epithelial cells and suggests shared pathogenetic pathways between COPD and the chronic airway disease cystic fibrosis. Similar to another common chronic airway disease, asthma, COPD airways exhibit increased VEGF and IL-13 levels, which contribute to mucous gland hyperplasia and bronchial smooth muscle hyperreactivity (67). It is interesting to note that the chronic bronchitis phenotype of COPD in humans is characterized by increased VEGF in the large airways, while in emphysema there is a paucity of VEGF in the lung parenchyma (6-8). Moreover, the small airways in COPD appear to exhibit unique remodeling mechanisms. Indeed, inhibition of EMAPII attenuates small airway but not large airway remodeling in mice chronically exposed to cigarette smoke (36). Furthermore, both TGF- $\beta$ and IL- $1 \beta$ were proposed as important mediators of airway remodeling in COPD explants (68). Recent studies revealed that small airways may disappear even earlier than surrounding alveoli (30), providing a common phenotype with that resulting from destructive processes occurring in emphysema. The mechanistic role of TGF- $\beta$ initially suggested in ex vivo models (68) has been recently supported by the beneficial effects of anti-TGF- $\beta$ anti- bodies or TGF- $\beta$ inhibition with the angiotensin receptor inhibitor losartan on both airway remodeling and airspace enlargement due to cigarette smoke (69). Similarly, both molecular and pharmacological inhibition of IL-1 $\beta$ signaling attenuated airway remodeling and extracellular hyaluronan levels during cigarette smoke exposure (70).

Lung cancer. There is evidence of both active cell proliferation and apoptosis in COPD lungs, and the balance of cell proliferation to death may be a measure of (inadequate) repair in emphysema (71). However, a proliferative response in airway epithelial cells exposed to cigarette smoke (72) may be unwanted. When combined with the cancer-initiation properties of tobacco, these events may underlie the cancer-promoting effects of cigarette smoke-induced inflammation (73). Accumulation of macromolecular damage in the course of COPD might also explain the increasingly recognized association between lung cancer and emphysema (74).

Systemic manifestations. The lung-specific pathogenetic processes outlined above may apply to systemic organ dysfunction, as the lung allows passage of oxidants into the bloodstream (75). Right ventricular dysfunction due to pulmonary hypertension may occur due to oxidative and nitrosative stress, with reduced expression of vasodilators in COPD (12, 76-78). Prolonged exposures to cigarette smoke have an inhibitory effect on the bone marrow hematopoietic progenitor cell number and cycling function $(79,80)$, adversely impacting lung repair mechanisms in COPD. Finally, skeletal muscle wasting and decreased physical activity, which are major comorbidities in COPD, have been linked to both increased apoptosis and decreased vascular regeneration $(81,82)$.

\section{Consolidation}

COPD may progress in patients despite smoking cessation, which challenges the concept of a direct link between ongoing exposure to cigarette smoke and the disease. This progression parallels persisting inflammatory responses (83), suggesting that additional mechanisms must account for the consolidation of the disease in genetically susceptible hosts, often after decades of active smoking (Figure 1). This change in the nature of inflammation in the course of disease is highlighted by the temporary nature of NF- $\mathrm{\kappa B}$ activation in rodent lungs exposed to cigarette smoke (84). Two paradigms have emerged that might explain some of these observations: autoimmunity (52) and lung aging (85). These findings may derive from the profound lung alveolar damage (39) and airway remodeling imposed by chronic cigarette smoke exposure and bombardment by endogenous mediators of inflammation and cell injury. Understanding the mechanism of this persistence might have a far-reaching impact on the design and implementation of regenerative therapies.

It is conceivable that COPD becomes over time an autoinflammatory disease, possibly involving the inflammasome and related cytokines. Cigarette smoke activates the inflammasome in mouse lungs, involving ATP and its receptor P2X7 purinergic receptor (86). NLPR-3 activation of caspase-1 (87) would lead to increased IL-1 $\beta$ (70) and IL-18 (62), both of which have been shown to participate in experimental models of cigarette smoke-induced inflammation and alveolar injury. Although DAMPs, including purines, can act early, in the initiation stage of COPD, the pathogenic roles of IL-18/IL-18 receptor signaling suggest that this inflammatory signaling occurs downstream of apoptosis, positioning it in the consolidation stage of alveolar injury (62). 
There is growing evidence for a role of autoreactive $\mathrm{T}$ cells or auto-antibodies in the activation of specific immunity in COPD. Mice exposed to cigarette smoke exhibit lung infiltration by CD8 $\mathrm{T}$ cell oligoclones (88), a finding that correlates with observations of oligoclones of CD4 cells in lungs of patients with COPD (58). Auto-antibodies have been detected in lung tissues of patients with advanced disease (89), including antibodies targeted to immunogenic carbonylated proteins (90). The latter findings were duplicated in mice exposed to cigarette smoke (91). Furthermore, rats immunized with endothelial cells developed autoreactive antibodies and $\mathrm{T}$ cells, which mediated alveolar enlargement (92). Finally, elastin-reactive T cells have been detected in the peripheral blood of patients with established COPD (93). Although the precise nature of the auto-reactive antigens will require further study, there is evidence of profound alterations in the structure of the lung during the course of exposure to cigarette smoke, ultimately leading to replacement of the alveolar elastin framework by collagen (94). A shared theme with other autoimmune processes is the emergence of TH17 lymphocytes, which are found in increased numbers in COPD lungs (95). Their potential role in alveolar destruction was recently uncovered by the protection against inflammation and emphysema observed in IL-17RA-knockout mice (96).

The relentless lung injury due to oxidant exposure, along with the potential exhaustion of lung protective responses, ultimately leads to lung aging, with increased expression of markers of cellular senescence (97). Again, a common denominator for aging and cellular senescence is oxidative stress, resulting in macromolecular damage, including the increased expression of markers of DNA damage $(39,98)$ and adduct-modified proteins $(99)$. The ultimate "biological clock" of cell turnover is controlled by telomerase, which preserves the shortening of ends of chromosomes during every round of mitosis. Lungs of patients with advanced emphysema have decreased telomere lengths in alveolar cells (97), which are paralleled by decreased telomere lengths in peripheral blood mononuclear cells $(100,101)$. The contribution of shortened telomeres was recently unraveled in investigations with the telomerase reverse transcriptase-knockout mice, which showed increased sensitivity to alveolar injury and airspace enlargement due to cigarette smoke, notably in late intercrosses compared with early intercrosses and wild-type mice (102).

\section{The future}

Key questions remain in our understanding of COPD, and these concern clinical phenotypes, systemic manifestations of COPD, and the impact of exacerbations triggered by infections. These central clinical manifestations of COPD probably result from the interaction of disease-related genes with fundamental processes involving inflammation, thrombosis and hemorrhage, fibrosis, the immune response, proliferation, and apoptosis/necrosis, which underlie the so-called intermediate phenotypes (103). Development of model systems that can address mechanistically these interactions will remain vital for progress in COPD, validated by studies of the human disease. Some therapeutic strategies, such as restoring $\alpha 1$-antitrypsin activity or the use of Nrf2-dependent antioxidants, may directly antagonize destructive processes such as the activation of pro-apoptotic mediators and extracellular matrix proteolysis $(36,104)$; a significant challenge lies in restoring lung survival mechanisms without fueling oncogenesis. Recently described shortcomings in attempts to regenerate the lung in a murine model of COPD (105) remind us that approaches aimed at lung organ restoration (79) will require consideration of the extent of macromolecular damage imposed by decades of lung destruction $(39,98)$. The aggregate of these insights into the pathogenesis of COPD provide landmarks that should direct future investigations in COPD and targets for potential novel therapies.

\section{Acknowledgments}

This work was supported by NIH grants ES016285 (to R.M. Tuder), HL077328 and HL090950 (to I. Petrache), and P50 HL084945 (Project 1, to R.M. Tuder and I. Petrache), a FAMRI research award (to R.M. Tuder), and a Veterans Health Administration merit award (to I. Petrache). The authors acknowledge the input of Alvar Agusti and Bartolome R. Celli regarding the current clinical challenges in COPD and Eric Schmidt and Laura Davis for proofreading this Review.

Address correspondence to: Rubin M. Tuder, Program in Translational Lung Research, Division of Pulmonary Sciences and Critical Care Medicine, Department of Medicine, University of Colorado at Denver, School of Medicine, 12700 East 19th Avenue, Research Complex 2, Aurora, Colorado, USA. Phone: 303.724.6062; Fax: 303.724.6042; E-mail: Rubin.Tuder@ucdenver.edu.
1. Rosenbaum L, Lamas D. Facing a "slow-motion disaster"-the UN meeting on noncommunicable diseases. N Engl J Med. 2011;365(25):2345-2348.

2. Han MK, et al. Chronic obstructive pulmonary disease phenotypes: the future of COPD. Am J Respir Crit Care Med. 2010;182(5):598-604.

3. MacNee W. Oxidants/antioxidants and chronic obstructive pulmonary disease: pathogenesis to therapy. Novartis Found Symp. 2001;234:169-185.

4. Eiserich JP, van der Vliet A, Handelman GJ, Halliwell B, Cross CE. Dietary antioxidants and cigarette smoke-induced biomolecular damage: a complex interaction. Am J Clin Nutr. 1995; 62(6 suppl):1490S-1500S.

5. MacNee W, Wiggs B, Belzberg AS, Hogg JC. The effect of cigarette smoking on neutrophil kinetics in human lungs. NEnglJ Med. 1989;321(14):924-928.

6. Yoshida T, et al. Rtp801, a suppressor of mTOR signaling, is an essential mediator of cigarette smokeinduced pulmonary injury and emphysema. Nat Med. 2010;16(7):767-773.

7. Schweitzer KS, et al. Mechanisms of lung endothelial barrier disruption induced by cigarette smoke: role of oxidative stress and ceramides. Am J Physiol Lung Cell Mol Physiol. 2011;301(6):L836-L846. 8. Presson RG Jr, et al. Two-photon imaging within the murine thorax without respiratory and cardiac motion artifact. Am J Pathol. 2011;179(1):75-82.

9. Rahman I, Adcock IM. Oxidative stress and redox regulation of lung inflammation in COPD. Eur Respir J. 2006;28(1):219-242.

10. Yoshida T, Tuder RM. Pathobiology of cigarette smoke-induced chronic obstructive pulmonary disease. Physiol Rev. 2007;87(3):1047-1082.

11. Rajendrasozhan S, Chung S, Sundar IK, Yao H, Rahman I. Targeted disruption of NF-\{kappa\} B1 (p50) augments cigarette smoke-induced lung inflammation and emphysema in mice: a critical role of p50 in chromatin remodeling. Am J Physiol Lung Cell Mol Physiol. 2010;298(2):L197-L209.

12. Seimetz M, et al. Inducible NOS inhibition reverses tobacco-smoke-induced emphysema and pulmonary hypertension in mice. Cell. 2011;147(2):293-305.

13. Weathington NM, et al. A novel peptide CXCR ligand derived from extracellular matrix degradation during airway inflammation. Nat Med.
2006;12(3):317-323.

14. Sarir H, Henricks PAJ, van Houwelingen $\mathrm{AH}$, Nijkamp FP, Folkerts G. Cells, mediators and Toll-like receptors in COPD. EurJ Pharmacol. 2008; 585(2-3):346-353.

15. Zhang X, Shan P, Jiang G, Cohn L, Lee PJ. Toll-like receptor 4 deficiency causes pulmonary emphysema. J Clin Invest. 2006;116(11):3050-3059.

16. Ellisen LW, et al. REDD1, a developmentally regulated transcriptional target of p63 and p53, links p63 to regulation of reactive oxygen species. $\mathrm{Mol}$ Cell. 2002;10(5):995-1005.

17. Shoshani $\mathrm{T}$, et al. Identification of a novel hypoxia-inducible factor 1-responsive gene, RTP801, involved in apoptosis. Mol Cell Biol. 2002; 22(7):2283-2293

18. Brugarolas J, et al. Regulation of mTOR function in response to hypoxia by REDD1 and the TSC1/ TSC2 tumor suppressor complex. Genes Dev. 2004; 18(23):2893-2904.

19. Malhotra D, et al. Global mapping of binding sites for Nrf2 identifies novel targets in cell survival response through ChIP-Seq profiling and network 
analysis. Nucleic Acids Res. 2010;38(17):5718-5734.

20. Rangasamy T, et al. Genetic ablation of Nrf2 enhances susceptibility to cigarette smoke-induced emphysema in mice. JClin Invest. 2004;114(9):1248-1259.

21. Eriksson S. Studies in alpha-1-atitrypsin. Acta Med Scand Suppl. 1965;432:1-85.

22. Kuhn C, Yu SY, Chraplyvy M, Linder HE, Senior RM. The induction of emphysema with elastase. II. Changes in connective tissue. Lab Invest. 1976; 34(4):372-380.

23. Hautamaki RD, Kobayashi DK, Senior RM, Shapiro SD. Requirement for macrophage elastase for cigarette smoke-induced emphysema in mice. Science. 1997;277(5334):2002-2004.

24. Tuder RM, Voelkel NF. Pathobiology of chronic bronchitis and emphysema. In: Voelkel NF, MacNee W, eds. Chronic Obstructive Lung Disease. Hamilton, Ontario, Canada: BC Decker Inc.; 2001:63-76.

25. Taraseviciene-Stewart L, Voelkel NF. Molecular pathogenesis of emphysema. J Clin Invest. 2008; 118(2):394-402.

26. Tuder RM, McGrath S, Neptune E. The pathobiological mechanisms of emphysema models: what do they have in common? Pulm Pharmacol Ther. 2003; 16(2):67-78.

27. Kasahara Y, et al. Inhibition of vascular endothelial growth factor receptors causes lung cell apoptosis and emphysema. JClin Invest. 2000;106(11):1311-1319.

28. Lam AP, Gottardi CJ, Tuder R. Regenerative pathways and emphysema: a new paradigm? Am J Respir Crit Care Med. 2011;183(6):688-690.

29. Nakanishi K, et al. Involvement of endothelial apoptosis underlying chronic obstructive pulmonary disease-like phenotype in adiponectin-null mice: implications for therapy. Am J Respir Crit Care Med. 2011;183(9):1164-1175.

30. McDonough JE, et al. Small-airway obstruction and emphysema in chronic obstructive pulmonary disease. N Engl J Med. 2011;365(17):1567-1575.

31. Tuder RM, Petrache I, Elias JA, Voelkel NF, Henson PM. Apoptosis and emphysema: the missing link. Am J Resp Cell Mol Biol. 2003;28(5):551-554.

32. Kasahara Y, Tuder RM, Cool CD, Lynch DA, Flores SC, Voelkel NF. Endothelial cell death and decreased expression of vascular endothelial growth factor and vascular endothelial growth factor receptor 2 in emphysema. Am J Respir Crit Care Med. 2001;163(3 pt 1):737-744.

33. Petrache I, et al. Ceramide upregulation causes pulmonary cell apoptosis and emphysema-like disease in mice. Nat Med. 2005;11(5):491-498.

34. Aoshiba K, Yokohori N, Nagai A. Alveolar wall apoptosis causes lung destruction and emphysematous changes. Am J Respir Cell Mol Biol. 2003;28(5):555-562.

35. Petrache I, et al. Superoxide dismutase protects against apoptosis and alveolar enlargement induced by ceramide. Am J Physiol Lung Cell Mol Physiol. 2008;295(1):L44-L53.

36. Clauss $M$, et al. Lung endothelial monocyte-activating protein 2 is a mediator of cigarette smokeinduced emphysema in mice. J Clin Invest. 2011; 121(6):2470-2479

37. Giordano RJ, Edwards JK, Tuder RM, Arap W, Pasqualini R. Combinatorial ligand-directed lung targeting. Proc Am Thorac Soc. 2009;6(5):411-415.

38. Malhotra D, et al. Heightened endoplasmic reticulum stress in COPD lungs: The role of Nrf2-regulated proteasomal activity. Am J Respir Crit Care Med. 2009;180(12):1196-1207.

39. Deslee $G$, et al. Oxidative damage to nucleic acids in severe emphysema. Chest. 2009;135(4):965-974.

40. Monick MM, et al. Identification of an autophagy defect in smokers' alveolar macrophages. J Immunol. 2010;185(9):5425-5435.

41. Petrusca DN, et al. Sphingolipid-mediated inhibition of apoptotic cell clearance by alveolar macrophages. J Biol Chem. 2010;285(51):40322-40332.

42. Monick MM, et al. Cooperative prosurvival activity by ERK and Akt in human alveolar macrophages is dependent on high levels of acid ceramidase activity. J Immunol. 2004;173(1):123-135.

43. Chen $\mathrm{ZH}$, et al. Egr-1 regulates autophagy in cigarette smoke-induced chronic obstructive pulmonary disease. PLoS ONE. 2008;3(10):e3316.

44. Csordas A, et al. Cigarette smoke extract induces prolonged endoplasmic reticulum stress and autophagic cell death in human umbilical vein endothelial cells. Cardiovasc Res. 2011;92(1):141-148.

45. Hwang JW, et al. Cigarette smoke-induced autophagy is regulated by SIRT1-PARP-1-dependent mechanism: implication in pathogenesis of COPD. Arch Biochem Biophys. 2010;500(2):203-209.

46. Kim HP, et al. Autophagic proteins regulate cigarette smoke-induced apoptosis: protective role of heme oxygenase-1. Autophagy. 2008;4(7):887-895.

47. Hogg JC. Why does airway inflammation persist after the smoking stops? Thorax. 2006;61(2):96-97.

48. Filosto $S$, et al. Neutral sphingomyelinase 2: a novel target in cigarette smoke-induced apoptosis and lung injury. Am J Respir Cell Mol Biol. 2011; 44(3):350-360.

49. Zheng T, et al. Role of cathepsin S-dependent epithelial cell apoptosis in IFN- $\gamma$-induced alveolar remodeling and pulmonary emphysema. J Immunol. 2005;174(12):8106-8115.

50. Tuder RM, et al. Oxidative stress and apoptosis interact and cause emphysema due to vascular endothelial growth factor receptor blockade. Am J Respir Cell Mol Biol. 2003;29(1):88-97.

51. Richens TR, et al. Cigarette smoke impairs clearance of apoptotic cells through oxidant-dependent activation of RhoA. Am J Respir Crit Care Med. 2009; 179(11):1011-1021.

52. Cosio MG, Saetta M, Agusti A. Immunologic aspects of chronic obstructive pulmonary disease. NEngl J Med. 2009;360(23):2445-2454.

53. Motz GT, Eppert BL, Wesselkamper SC, Flury JL, Borchers MT. Chronic cigarette smoke exposure generates pathogenic $\mathrm{T}$ cells capable of driving COPD-like disease in Rag2 $2^{-/-}$mice. Am J Respir Crit Care Med. 2010;181(11):1223-1233.

54. Ito K, et al. Decreased histone deacetylase activity in chronic obstructive pulmonary disease. $N$ Engl J Med. 2005;352(19):1967-1976.

55. Rajendrasozhan S, Yang SR, Kinnula VL, Rahman I. SIRT1, an antiinflammatory and antiaging protein, is decreased in lungs of patients with chronic obstructive pulmonary disease. Am J Respir Crit Care Med. 2008;177(8):861-870.

56. Shaykhiev R, et al. Smoking-dependent reprogramming of alveolar macrophage polarization: implication for pathogenesis of chronic obstructive pulmonary disease. J Immunol. 2009; 183(4):2867-2883

57. Majo J, Ghezzo H, Cosio MG. Lymphocyte population and apoptosis in the lungs of smokers and their relation to emphysema. Eur Respir J. 2001; 17(5):946-953.

58. Sullivan AK, et al. Oligoclonal CD4+ T cells in the lungs of patients with severe emphysema. Am J Respir Crit Care Med. 2005;172(5):590-596.

59. Borchers MT, et al. Sustained CTL activation by murine pulmonary epithelial cells promotes the development of COPD-like disease. J Clin Invest. 2009;119(3):636-649.

60. Malhotra D, et al. Decline in NRF2-regulated antioxidants in chronic obstructive pulmonary disease lungs due to loss of Its positive regulator, DJ-1. Am J Respir Crit Care Med. 2008;178(6):592-604.

61 . Harvey CJ, et al. Targeting Nrf2 signaling improves bacterial clearance by alveolar macrophages in patients with COPD and in a mouse model. Sci Transl Med. 2011;3(78):78ra32.

62. Kang MJ, et al. Cigarette smoke selectively enhances viral PAMP and viruses-induced pulmonary innate immunity and remodeling responses. J Clin
Invest. 2008;118(8):2771-2784.

63. Ferhani N, et al. Expression of high-mobility group box 1 and of receptor for advanced glycation end products in chronic obstructive pulmonary disease. Am J Respir Crit Care Med. 2010;181(9):917-927.

64. Houghton AM, et al. Elastin fragments drive disease progression in a murine model of emphysema. J Clin Invest. 2006;116(3):753-759.

65. Hogg J. Peripheral lung remodelling in asthma and chronic obstructive pulmonary disease. Eur RespirJ. 2004;24(6):893-894

66. Cantin AM, et al. Cystic fibrosis transmembrane conductance regulator function is suppressed in cigarette smokers. Am J Respir Crit Care Med. 2006; 173(10):1139-1144.

67. Lee CG, et al. Studies of vascular endothelial growth factor in asthma and chronic obstructive pulmonary disease. Proc Am Thorac Soc. 2011; 8(6):512-515.

68. Wang RD, Wright JL, Churg A. Transforming growth factor-beta1 drives airway remodeling in cigarette smoke-exposed tracheal explants. Am J Respir Cell Mol Biol. 2005;33(4):387-393.

69. Podowski M, et al. Angiotensin receptor blockade attenuates cigarette smoke induced lung injury and rescues lung architecture in a murine model. J Clin Invest. 2011;122(1):229-240.

70. Churg A, Zhou S, Wang X, Wang R, Wright JL. The role of interleukin-1beta in murine cigarette smoke-induced emphysema and small airway remodeling. Am J Respir Cell Mol Biol. 2009; 40(4):482-490.

71. Imai K, Mercer BA, Schulman LL, Sonett JR, D'Armiento JM. Correlation of lung surface area to apoptosis and proliferation in human emphysema. Eur Respir J. 2005;25(2):250-258.

72. Lemjabbar H, Li D, Gallup M, Sidhu S, Drori E, Basbaum C. Tobacco smoke-induced lung cell proliferation mediated by tumor necrosis factor alphaconverting enzyme and amphiregulin. J Biol Chem. 2003;278(28):26202-26207.

73. Takahashi H, Ogata H, Nishigaki R, Broide DH, Karin M. Tobacco smoke promotes lung tumorigenesis by triggering IKKbeta- and JNK1-dependent inflammation. Cancer Cell. 2010;17(1):89-97.

74. Punturieri A, Szabo E, Croxton TL, Shapiro SD, Dubinett SM. Lung cancer and chronic obstructive pulmonary disease: needs and opportunities for integrated research. J Natl Cancer Inst. 2009; 101(8):554-559

75. Yamaguchi Y, Nasu F, Harada A, Kunitomo M. Oxidants in the gas phase of cigarette smoke pass through the lung alveolar wall and raise systemic oxidative stress. J Pharmacol Sci. 2007;103(3):275-282.

76. Sussan TE, et al. Targeting Nrf2 with the triterpenoid CDDO-imidazolide attenuates cigarette smoke-induced emphysema and cardiac dysfunction in mice. Proc Natl Acad Sci U S A. 2009; 106(1):250-255

77. Santos S, et al. Characterization of pulmonary vascular remodelling in smokers and patients with mild COPD. Eur Respir J. 2002;19(4):632-638.

78. Wright JL, Churg A. Short-term exposure to cigarette smoke induces endothelial dysfunction in small intrapulmonary arteries: analysis using guinea pig precision cut lung slices. J Appl Physiol. 2008;104(5):1462-1469.

79. Schweitzer KS, et al. Adipose stem cell treatment in mice attenuates lung and systemic injury induced by cigarette smoking. Am J Respir Crit Care Med. 2011; 183(2):215-225

80. Palange $\mathrm{P}$, et al. Circulating haemopoietic and endothelial progenitor cells are decreased in COPD. Eur Respir J. 2006;27(3):529-541.

81. Agusti AG, et al. Skeletal muscle apoptosis and weight loss in chronic obstructive pulmonary disease. Am J Respir Crit Care Med. 2002;166(4):485-489.

82. Langen RC, Schols AM, Kelders MC, van der Velden 
JL, Wouters EF, Janssen-Heininger YM. Muscle wasting and impaired muscle regeneration in a murine model of chronic pulmonary inflammation. Am J Respir Cell Mol Biol. 2006;35(6):689-696.

83. Willemse BW, ten Hacken NH, Rutgers B, LesmanLeegte IG, Postma DS, Timens W. Effect of 1-year smoking cessation on airway inflammation in COPD and asymptomatic smokers. Eur Respir J. 2005;26(5):835-845.

84. Moriyama C, et al. Aging enhances susceptibility to cigarette smoke-induced inflammation through bronchiolar chemokines. Am J Respir Cell Mol Biol. 2010;42(3):304-311.

85. Tuder RM, Yoshida T, Arap W, Pasqualini R, Petrache I. Cellular and molecular mechanisms of alveolar destruction in emphysema: an evolutionary perspective. Proc Am Thorac Soc. 2006;3(6):503-510.

86. Lucattelli $\mathrm{M}$, et al. $\mathrm{P} 2 \mathrm{X} 7$ receptor signaling in the pathogenesis of smoke-induced lung inflammation and emphysema. Am J Respir Cell Mol Biol. 2011; 44(3):423-429.

87. Eltom S, et al. P2X7 receptor and caspase 1 activation are central to airway inflammation observed after exposure to tobacco smoke. PLoS One. 2011; 6(9):e24097.

88. Motz GT, et al. Persistence of lung CD8 T cell oligoclonal expansions upon smoking cessation in a mouse model of cigarette smoke-induced emphysema. J Immunol. 2008;181(11):8036-8043.
89. Feghali-Bostwick CA, et al. Autoantibodies in patients with chronic obstructive pulmonary disease. Am J Respir Crit Care Med. 2008;177(2):156-163.

90. Kirkham PA, et al. Oxidative stress-induced antibodies to carbonyl-modified protein correlate with severity of chronic obstructive pulmonary disease. Am J Respir Crit Care Med. 2011;184(7):796-802.

91. Brandsma CA, et al. Induction of autoantibodies against lung matrix proteins and smoke-induced inflammation in mice. BMC Pulm Med. 2010;10:64.

92. Taraseviciene-Stewart L, et al. An animal model of autoimmune emphysema. Am J Respir Crit Care Med. 2005;171(7):734-742.

93. Lee SH, et al. Antielastin autoimmunity in tobacco smoking-induced emphysema. Nat Med. 2007;13(5):567-569.

94. Vlahovic G, Russell ML, Mercer RR, Crapo JD. Cellular and connective tissue changes in alveolar septal walls in emphysema. Am J Respir Crit Care Med. 1999; 160(6):2086-2092.

95. Shan M, et al. Lung myeloid dendritic cells coordinately induce TH1 and TH17 responses in human emphysema. Sci Transl Med. 2009;1(4):4ra10.

96. Chen K, et al. IL-17RA is required for CCL2 expression, macrophage recruitment, and emphysema in response to cigarette smoke. PLoS One. 2011; 6(5):e20333.

97. Tsuji T, Aoshiba K, Nagai A. Alveolar cell senescence in pulmonary emphysema patients. Am J
Respir Crit Care Med. 2006;174(8):886-893.

98. Pastukh VM, et al. Oxidative DNA damage in lung tissue from patients with COPD is clustered in functionally significant sequences. Int J Chron Obstruct Pulmon Dis. 2011;6:209-217.

99. Rahman I, et al. 4-Hydroxy-2-Nonenal, a specific lipid peroxidation product, is elevated in lungs of patients with chronic obstructive pulmonary disease. Am J Respir Crit Care Med. 2002;166(4):490-495.

100. Morla M, Busquets X, Pons J, Sauleda J, MacNee W, Agusti AG. Telomere shortening in smokers with and without COPD. Eur Respir J. 2006;27(3):525-528.

101. Savale L, et al. Shortened telomeres in circulating leukocytes of patients with chronic obstructive pulmonary disease. Am J Respir Crit Care Med. 2009; 179(7):566-571.

102.Alder JK, et al. Telomere length is a determinant of emphysema susceptibility. Am J Respir Crit Care Med. 2011;184(8):904-912.

103.Agusti A, Vestbo J. Current controversies and future perspectives in chronic obstructive pulmonary disease. Am J Respir Crit Care Med. 2011;184(5):507-513.

104.Diab KJ, et al. Stimulation of sphingosine 1-phosphate signaling as an alveolar cell survival strategy in emphysema. Am J Respir Crit Care Med. 2010; 181(4):344-352.

105. Massaro GD, Massaro D. Retinoic acid treatment abrogates elastase-induced pulmonary emphysema in rats. Nat Med. 1997;3(6):675-677. 